\title{
Chemical enrichment and feedback in low metallicity environments: constraints on galaxy formation
}

\author{
Francesca Matteucci ${ }^{1,2}$ \\ ${ }^{1}$ Dipartimento di Astronomia, Trieste University \\ Via G.B. Tiepolo 11, 34131 Trieste, Italy \\ ${ }^{2}$ Italian National Institute for Astrophysics (INAF), Trieste \\ Via G.B. Tiepolo 11, 34131 Trieste, Italy \\ email: matteucc@oats.inaf.it
}

\begin{abstract}
Chemical evolution models for dwarf metal poor galaxies, including dwarf irregulars and dwarf spheroidals will be presented. The main ingredients necessary to build detailed models of chemical evolution including stellar nucleosynthesis, supernova progenitors, stellar lifetimes and stellar feedback will be discussed. The stellar feedback will be analysed in connection with the development of galactic winds in dwarf galaxies and their effects on the predicted abundances and abundance ratios. Model results concerning $\alpha$-elements $(\mathrm{O}, \mathrm{Mg}, \mathrm{Si}, \mathrm{Ca}), \mathrm{Fe}$ and s-and r-process elements will be discussed and compared with the most recent observational data for metal poor galaxies of the Local Group. We will show how the study of abundance ratios versus abundances can represent a very powerful tool to infer constraints on galaxy formation mechanisms. In this framework, we will discuss whether, on the basis of their chemical properties, the dwarf galaxies of the Local Group could have been the building blocks of the Milky Way.
\end{abstract}

Keywords. ISM: abundances, galaxies: abundances, galaxies: evolution

\section{Introduction}

Galactic chemical evolution studies how the abundances of the elements in the interstellar medium (ISM) evolve in space and time. In order to do that we need to build models containing important prescriptions relative to the most important physical processes acting in galaxies. In particular the main parameters are:

- The initial conditions, namely whether the gas which forms a galaxy has a primordial chemical composition or has been pre-enriched by an early stellar generation. Then one should decide whether the system is closed (no exchange of gas with the surrounding) or open (infall, outflow).

- The birthrate function is defined as the product of the star formation rate (SFR), $\psi(t)$ and the initial mass function (IMF), $\varphi(m)=I M F$. The SFR can be parametrized in several ways, such as exponentially decreasing in time with a typical timescale which should be fixed by reproducing the present time properties of the studied object. However, the most common parametrization of the SFR is the Schmidt (1959) law where the SFR is proportional to some power $(k=1-2)$ of the surface gas density:

$$
S F R=\nu \sigma_{g a s}^{k}
$$

where $\nu$ is the efficiency of star formation, namely the inverse of the star formation timescale and it is fixed by reproducing the present time SFR. 
The IMF is a probability function and is normally described by a power law with one or more slopes:

$$
\varphi(M)=a M^{-(1+x)}
$$

and is the number of stars with masses in the interval $M, M+d M$.

The IMF has been derived only for the stars in the solar vicinity and we recall the one of Salpeter (1955) with only one slope $x=1.35$ for stars $>10 M_{\odot}$ and the more recent ones, extended to low mass stars with more than one slope (Scalo 1986, 1998; Kroupa et al. 1993). We do not have any information about the IMF in other galaxies than the Milky Way.

- Stellar evolution and nucleosynthesis, in other words the stellar yields. Stars contribute in a different way to the galactic chemical enrichment according to their mass:

-Low and Intermediate mass stars $\left(0.8 \leqslant M / M_{\odot} \leqslant 8.0\right)$ produce ${ }^{4} \mathrm{He}, \mathrm{C}, \mathrm{N}$ (primary and secondary) and s-process $(A>90)$ elements.

-Massive stars $\left(M \geqslant 10 M_{\odot}\right)$ which die as core-collapse $\mathrm{SNe}(\mathrm{SNe}$ II and $\mathrm{SNe} \mathrm{Ib} / \mathrm{c}$ ) produce mainly $\alpha$-elements $(\mathrm{O}, \mathrm{Ne}, \mathrm{Mg}, \mathrm{Si}, \mathrm{S}, \mathrm{Ca})$, some Fe-peak elements, s-process elements $(A<90)$ and r-process elements during the $\mathrm{SN}$ explosion.

-White dwarfs (WD) of $\mathrm{C}$ and $\mathrm{O}$ (therefore originating in the low and intermediate mass range) in binary systems can give rise to Type Ia SNe which produce mainly $\mathrm{Fe}$ and Fe-peak elements. The most common scenarios for the progenitors of these SNe are: i) the Single Degenerate (SD) scenario and ii) the Double Degenerate (DD) scenario. The SD scenario, namely the classical scenario of Whelan and Iben (1973), consists in the C-deflagration in a C-O WD reaching the Chandrasekhar mass, $M_{C h}$, after accreting material from a red giant companion. The minimum time scale for the first explosion to occur in this scenario is $t_{S N I a_{m} \text { in }}=0.03$ Gyr (Greggio and Renzini 1983; Matteucci \& Recchi 2001) and the maximum is as long as a Hubble time. The DD scenario consists in the merging of two C-O WDs, due to gravitational wave radiation, which explode by C-deflagration when $M_{C h}$ is reached (Iben and Tutukov 1984). The minimum timescale for the first explosion here is only slightly longer $\sim 0.031$ Gyr (Greggio 2005), while the maximum timescale can be several Hubble times. Recently an empirical bimodal SN Ia rate (Mannucci et al. 2005;2006) has been proposed: $\sim 50 \%$ of all Type Ia SNe arise from binary systems with lifetimes $<10^{8}$ years, whereas the rest comes from smaller progenitors with a much broader distribution of stellar lifetimes (see also Matteucci et al. 2006).

-Very Massive Objects (VMO) $\left(M>100 M_{\odot}\right.$, Pop III), if they ever existed, could form only up to a metallicity of $\left(10^{-6}-10^{-4}\right) Z_{\odot}$ (Schneider et al. 2006) and therefore their effect on the element production was negligible (Ballero et al. 2006).

- Gas infall and/or outflow from the system. For the infall rate (IR) normally an exponential function is assumed:

$$
I R=C e^{-t / \tau}
$$

where $\tau$ is a typical timescale. For the galactic wind the most common parametrization is to adopt that the wind rate (WR) is several times the star formation rate:

$$
W R=\lambda S F R \text {. }
$$

The constants $C$ and $\lambda$ are suitable parameters chosen to reproduce the present time properties of the studied galaxies.

Chemodynamical models of dwarf galaxies have shown that galactic winds are easily produced in these galaxies and that they are metal-enriched (e.g. McLow \& Ferrara, 1999; D'Ercole \& Brighenti 1999; Recchi et al. 2001). 


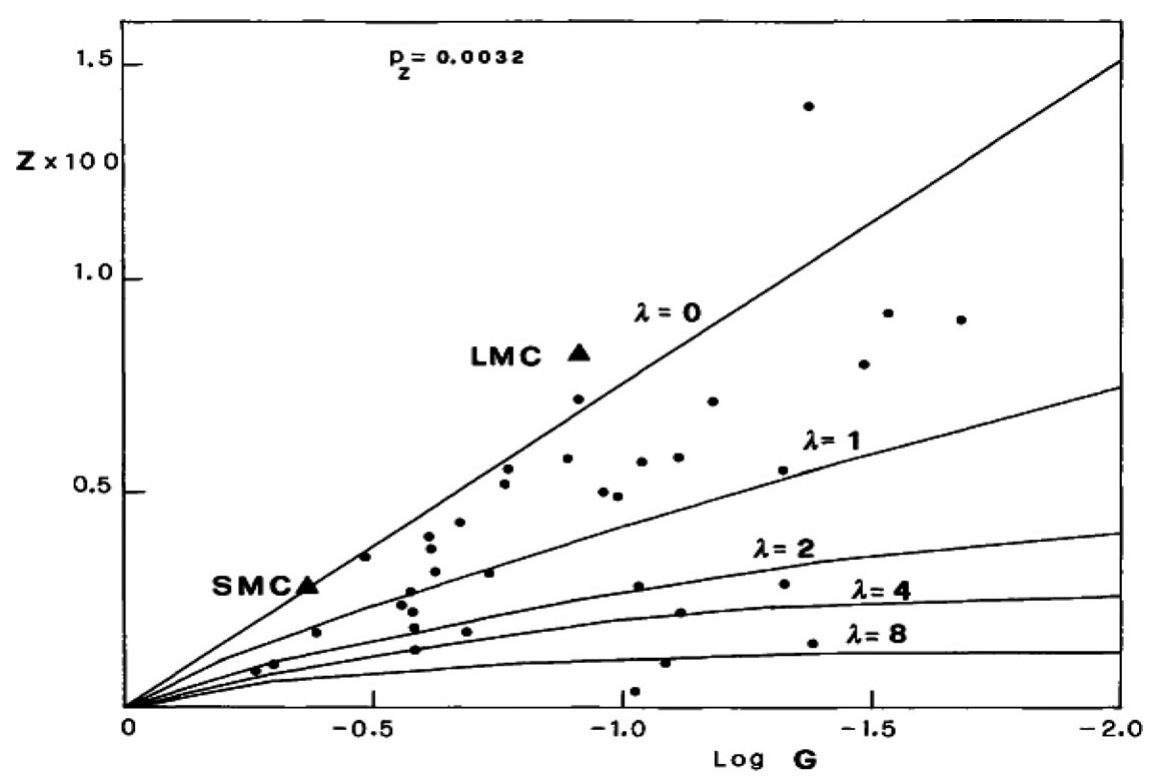

Figure 1. The Z- $\log G$ diagram. Solution b.)(see text) from Matteucci \& Chiosi (1983). Note that the solution of the Simple Model $(\lambda=0)$ corresponds to the effective yield $p_{Z}$, which is the highest possible yield per stellar generation.

Here we will describe the chemical evolution of low metallicity dwarf galaxies, in particular of dwarf irregulars and blue compact galaxies where both the infall and outflow are taken into account.

\section{Dwarf Irregulars}

Dwarf Irregular (DIG) and Blue Compact galaxies (BCG) are important objects where to test galaxy evolution. They have a low metal content (abundances measured from HII regions) and large gas fractions suggesting that they either suffered isolated bursts of star formation and/or a low but continuous star formation (Searle et al. 1973). Both DIG and BCG show a distinctive spread in their chemical properties, although this spread is decreasing with the new more accurate data, but also a definite mass-metallicity relation.

From the point of view of chemical evolution, Matteucci and Chiosi (1983) first studied the evolution of DIG and BCG by means of analytical chemical evolution models including either outflow or infall and concluded that: closed-box models cannot account for the Z-log $\mathrm{G}\left(G=M_{\text {gas }} / M_{\text {tot }}\right)$ distribution even if the number of bursts varies from galaxy to galaxy and suggested possible solutions to explain the observed spread. The possible solutions suggested were: a.) different IMF's, b.) different rates of galactic wind and c.) different rates of infall. In Figure 1 we show graphically the solution b.) which refers to different rates of galactic wind obtained just by varying $\lambda$. Later on, Matteucci \& Tosi (1985) tried the three solutions in a detailed numerical model and concluded that the most likely solution is b.), on the basis of reproducing the majority of the observed features in these galaxies, including the mass-metallicity relation.

Later on, Pilyugin (1993) forwarded the idea that the observed spread could be due to self-pollution of the HII regions, which do not mix efficiently with the surrounding medium, coupled with "enriched" or "differential" galactic winds, namely different chemical elements are lost at different rates. Other models in the following years (Marconi et al. 1994; Kunth et al. 1995; Bradamante et al. 1998) followed the suggestions of differential 

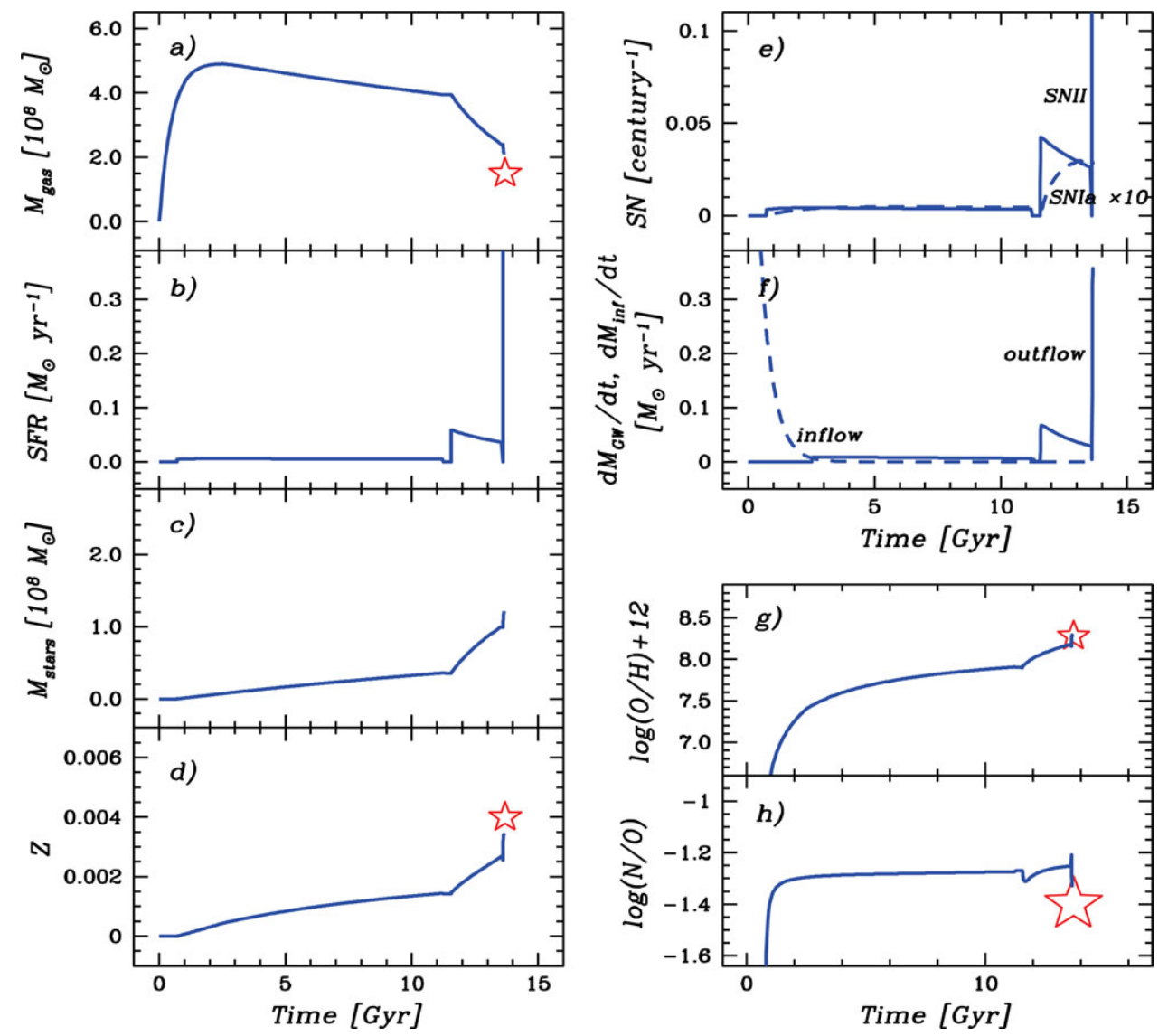

Figure 2. Comparison between data and model for NGC1569. The model (continuous lines) is from Romano et al. (2006), where the references to the data (stars) can also be found.

winds and introduced the novelty of the contribution to the chemical enrichment and energetics of the ISM by SNe of different Type (II, Ia and Ib).

\subsection{Galactic outflows}

Galactic outflows have been observed in some irregular dwarfs (e.g. Martin et al. 2002). More recently outflows have been observed with Chandra (Ott et al. 2005) in several star bursting dwarfs including IZw18 (see Aloisi this conference) and NGC1569 (see later). However, it is not entirely clear whether the outflowing material will eventually be lost from the potential well of the galaxy (wind) or it will cool and recollapse back into the galaxy (galactic fountain).

In models of chemical evolution of dwarf irregulars (e.g. Bradamante et al. 1998) the feedback effects are taken into account and the condition for the development of a wind is:

$$
\left(E_{t h}\right)_{I S M} \geqslant E_{\text {Bgas }}
$$

namely, that the thermal energy of the gas is larger or equal to its binding energy.

The thermal energy of gas due to SN and stellar wind heating is:

$$
\left(E_{t h}\right)_{I S M}=E_{t h_{S N}}+E_{t h_{w}}
$$


but the contribution from stellar wind is negligible relative to that of SNe. Two parameters here play a fundamental role: the efficiency transfer of the SN energy and the stellar wind energy into the ISM. In other words, one has to assume that a fraction of the initial SN blast wave energy $\left(E_{o}=10^{51} \mathrm{erg}\right)$ is going to thermalize the ISM, and the same for stellar winds although they can be neglected. These efficiencies are unfortunately still largely unknown. In Bradamante et al. (1998) the SN energy transfer efficiency is assumed to be $3 \%$ but it depends on the physical conditions of the ISM and in some situation it can be $100 \%$ (see Recchi et al. 2001).

On the other hand, the binding energy of the gas, $E_{B g a s}(t)$, should be computed by assuming a dark matter halo. The typical model for a BCG has a luminous mass of $\left(10^{8}-10^{9}\right) M_{\odot}$, a dark matter halo ten times larger than the luminous mass and various values for the parameter describing the distribution of the dark matter halo (in the Bradamante et al. paper the dark matter is 10 times more diffuse than the luminous matter). It is found that the galactic wind in these galaxies develops easily but it carries out mainly metals so that the total mass lost in the wind is small. These results have been confirmed by more sophisticated chemo-dynamical models (see Recchi et al. 2001).

\subsection{A specific galaxy: NGC1569}

As an example we show the chemical evolution results for a local dwarf irregular NGC1569, which has been observed with the Hubble Space Telescope (HST) and its color-magnitude diagram derived. From this color-magnitude diagram it has been inferred the star formation history of this object, in particular that it suffered two major bursts in the last 2 Gyrs and low but continuous star formation before (Angeretti et al. 2005). Gas outflows have also been observed (Ott et al. 2005). Romano et al. (2006) modeled the chemical evolution of NGC1569 following the SFR suggested by the color-magnitude diagram. They assumed that the rate of mass outflow is proportional to the Type II and Ia SN

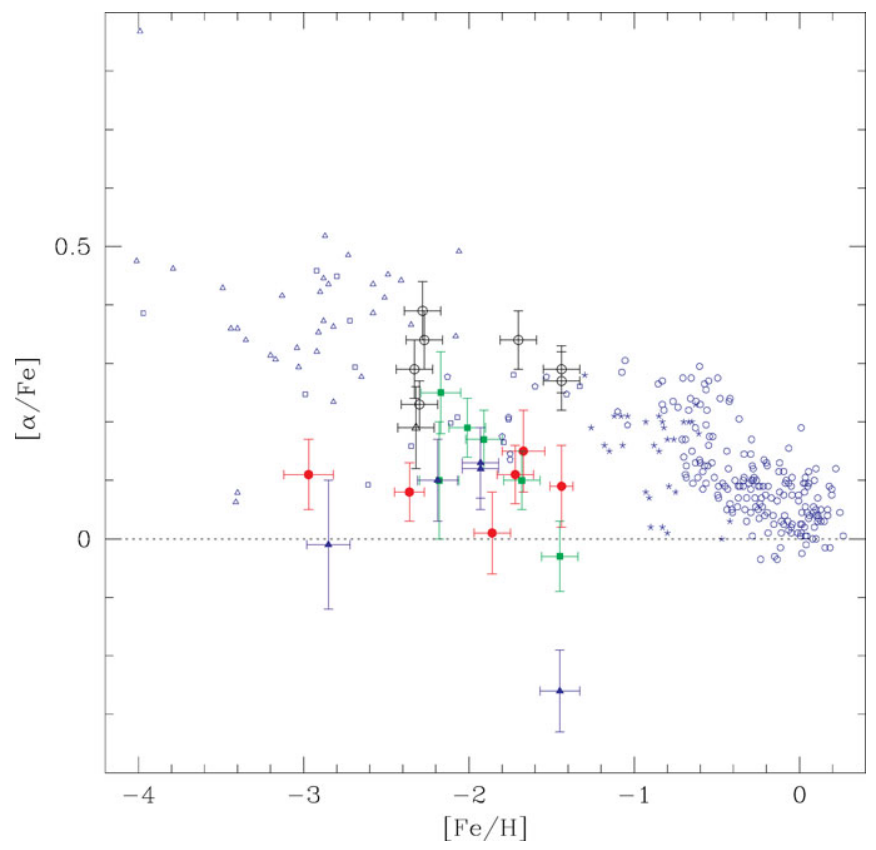

Figure 3. Comparison of $[\alpha / \mathrm{Fe}]$ ratios in dSphs and in the Milky Way: figure from Shetrone et al. (2003). The blue dots represent Galactic stars whereas the others are the dSphs. 
rates instead than to the SFR. This implies a more realistic treatment of the outflow relative to previous models where the wind stops when the SF stops. In this case, instead, the winds continue due to the energetic input of Type Ia SNe. In figure 2 we show the results for NGC1569 compared with the observations.

\section{Dwarf Spheroidals}

Dwarf spheroidal galaxies (dSphs) are small systems surrounding the Milky Way which are often indicated as the building blocks of the Galaxy in the framework of a hierarchical growth of structures. It is interesting to compare the abundance ratios, recently measured in an accurate way also in these systems, with those of the Milky Way. In Figure 3 we

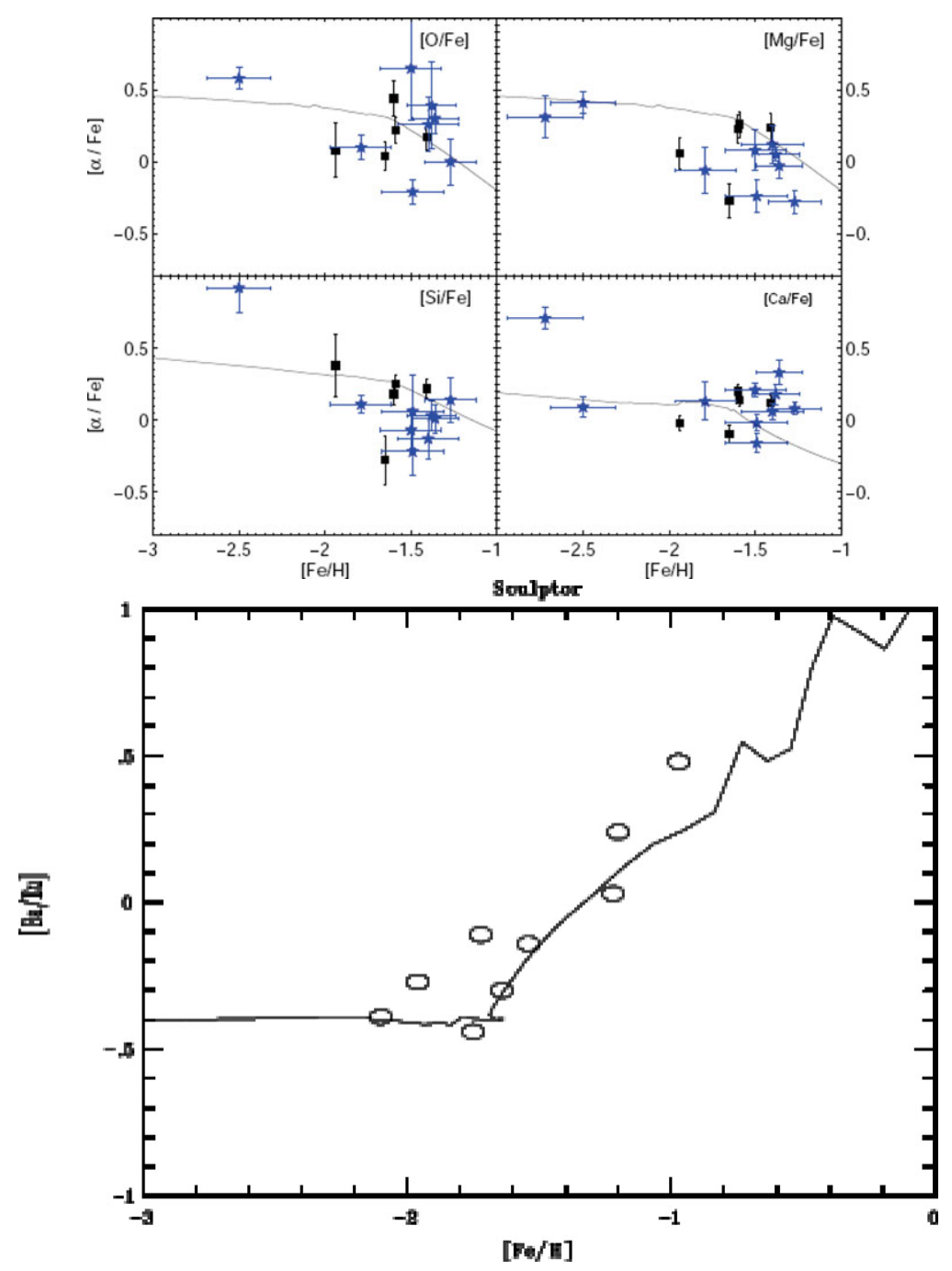

Figure 4. Upper figure: abundance ratios for the $\alpha$-elements $\mathrm{O}, \mathrm{Mg}, \mathrm{Si}$ and $\mathrm{Ca}$, as measured by Koch et al. (2008), shown as blue stars, in comparison with the model calculations from LMC06 (solid lines). Also plotted are the five data points from Shethrone et al. (2003) (black squares). Figure and the rest of data from Koch et al. (2008). Lower figure: measured and predicted $[\mathrm{Ba} / \mathrm{Eu}]$ ratios for the dSph Sculptor. The model is represented by the solid line and it is from LMC06. Figure and data from Geisler et al. (2007). 
show such a comparison, indicating that apart from a small overlap of the dSph data with the Galactic data at relatively low metallicities, the dSph data have a quite different behaviour, showing low $[\alpha / \mathrm{Fe}]$ ratios at low metallicities. This behaviour resembles the one predicted for systems with slow star formation (see Matteucci 2001). In fact, the time-delay with which the bulk of Fe is restored by Type Ia SNe, when combined with different star formation rates, produces different behaviours for the $[\alpha / \mathrm{Fe}]$ ratios: a fast star formation produces a longer plateau, whereas a slow star formation produces a smaller plateau and a steeper decline, relative to the solar vicinity. This induces to think that the different behaviour of the $[\alpha / \mathrm{Fe}]$ ratios in dSphs has something to do with their SF histories. To check this point Lanfranchi \& Matteucci (2004) and Lanfranchi et al. (2006, hereafter LMC06) have computed models for dSphs by adopting the SF histories suggested by the color-magnitude diagrams of these systems. Their models include also powerful galactic winds assumed to be proportional to the SFR, which devoid the dSphs of all the residual gas after star formation. As one can see in Figure 4, where model predictions and observations are shown, the model reproduces quite well the observations of Carina. This is due to the combination of a slow SFR and a strong wind which subtracts gas from the system thus further lowering the SFR. Always in Figure 4 we show the predictions of $\mathrm{LMC} 06$ for the $[\mathrm{Ba} / \mathrm{Eu}]$ ratio in Sculptor and the agreement is also quite good. Finally, LMC06 reproduced very well the stellar metallicity distribution in Carina including the lack of stars with $[\mathrm{Fe} / \mathrm{H}]<-3.0$ (Helmi et al. 2006): this is due to the assumption that these galaxies do not evolve as a closed box but infall played a role in the early stages of their formation.

\section{Conclusions}

We have described the evolution of low metallicity dwarfs, in particular dwarf irregulars and dwarf spheroidals.

Our main conclusions can be summarized as:

- The main properties of dwarf irregulars can be well reproduced with metal enhanced galactic winds. The winds carry out mostly metals, with a small net loss of gas. The star formation in these systems could have proceeded either in small bursts or at very low level but continuously with bursts overimposed.

- The dSphs instead must have suffered huge losses of gas, which lasted for several Gyrs, coupled with a low star formation efficiency. Low star formation and strong winds allow us to reproduce most of their observed abundance ratios ( $\alpha$ - and s- and r-process elements). DSphs seem to have evolved differently from the Milky Way, as shown by their different $[\alpha / \mathrm{Fe}]$ and $[\mathrm{s}, \mathrm{r} / \mathrm{Fe}]$ ratios.

- The observed lack of stars with $[\mathrm{Fe} / \mathrm{H}]<-3.0$ dex in dSphs can be well explained if these galaxies formed by infall of gas on a timescale of $\sim 0.5 \mathrm{Gyr}$. The problem is similar to the G-dwarf metallicity distribution in the solar vicinity.

- Objects similar to the local dwarf irregulars and dSphs are unlikely to have been the building blocks of the Milky Way.

\section{References}

Angeretti, L., Tosi, M., Greggio, L., Sabbi, E., Aloisi, A. \& Leitherer, Claus 2005, AJ, 129, 2203

Ballero, S.K., Matteucci, F. \& Chiappini, C. 2006, New Astr., 11, 306

Bradamante, F., Matteucci, F. \& D'Ercole, A. 1998, A\& $A$, 337, 338

D'Ercole, A. \& Brighenti, F. 1999, MNRAS, 309, 941

Geisler, D., Wallerstein, G., Smith, V. V. \& Casetti-Dinescu, D. I. 2007, PASP 119, 939 
Greggio, L. 2005, A\&A 441, 1055

Greggio, L. \& Renzini, A. 1983, A\&SA, 118, 217

Helmi, A., Irwin, M. J., Tolstoy, E., Battaglia, G., Hill, V., Jablonka, P., Venn, K., Shetrone, M., Letarte, B., Arimoto, N. et al. 2006, ApJ 651, L121

Iben, I. Jr. \& Tutukov, A. 1984, ApJ 284, 719

Koch, A. Grebel, E. K., Gilmore, G. F., Wyse, R. F. G., Kleyna, J. T., Harbeck, D. R., Wilkinson, M. I. \& Wyn Evans, N. 2008, AJ, 135, 1580

Kroupa, P., Tout, C.A. \& Gilmore, G. 1993, MNRAS 262, 545

Kunth, D., Matteucci, F. \& Marconi, G. 1995, A\& A, 297, 634

Lanfranchi, G. \& Matteucci, F. 2004, MNRAS, 351, 1338

Lanfranchi, G. \& Matteucci, F. \& Cescutti, G 2006, MNRAS, 365, 477 (LMC06)

Mac Low, M. \& Ferrara, A. 1999, ApJ, 513, 142

Mannucci, F., Della Valle, M., Panagia, N., Cappellaro, E., Cresci, G., Maiolino, R., Petrosian, A. \& Turatto, M. 2005, A\& $A 433,807$

Mannucci, F., Della Valle, M. \& Panagia, N. 2006, MNRAS, 370, 773

Marconi, G., Matteucci, F. \& Tosi, M. 1994, MNRAS, 270, 35

Martin, C. L., Kobulnicky, H. A. \& Heckman, T. M. 2002, ApJ, 574, 663

Matteucci, F. 2001, The Chemical Evolution of the Galaxy, ASSL, Kluwer Academic Publisher

Matteucci, F. \& Chiosi, C. 1983, A\&SA 123, 121

Matteucci, F. \& Recchi, S. 2001, ApJ 558, 351

Matteucci, F., Panagia, N., Pipino, A., Mannucci, F., Recchi, S. \& Della Valle, M., 2006 MNRAS, 372,265

Matteucci, F. \& Tosi, M. 1895, MNRAS, 217, 391

Ott, J., Walter, F., Brinks, E. \& al. 2005, MNRAS, 358, 1423

Pilyugin, I.S. 1993, A\&A 277, 42

Recchi, S., Matteucci, F. \& D'Ercole, A. 2001, MNRAS 322, 800

Romano, D., Tosi, M. \& Matteucci, F. 2006, MNRAS, 365, 759

Salpeter, E.E. 1955, ApJ 121, 161

Schmidt, M. 1959, ApJ 129, 243

Schneider, R., Salvaterra, R., Ferrara, A. \& Ciardi, B. 2006, MNRAS, 369, 825

Searle, L., Sargent, W. L. W. \& Bagnuolo, W. G. 1973, ApJ, 179, 427

Shetrone M., Venn K.A., Tolstoy E., Primas F. 2003, AJ, 125, 684

Whelan, J. \& Iben, I. Jr. 1973, ApJ 186, 1007 